\title{
UN NUEVO INDICADOR PARA LA HISTORIA FINANCIERA ESPAÑOLA: LA COTIZACION DE LAS LETRAS DE CAMBIO A CORTO PLAZO *
}

\author{
LLUIS CASTAÑEDA \\ (Instituto Universitario Europeo, Florencia) \\ XAVIER TAFUNELL \\ (Universitat Pompeu Fabra, Barcelona)
}

\begin{abstract}
RESUMEN
Con eata nota pretendemos mostrar una perspectiva inéditu del funcionamiento de los mencados financieros. Se trata de una primera reflexión sobre el precio de la movilidad del dinero, basada en las cotizaciones de las letrns de cambio a muy corto plavo sobre plazas nacionales. En la nota se expone la fuente utilizada, se desarrolla el manco conceptual que justifica considerar a los cambios como el precio de movilizar el dinero y, por último, se procede a un análisis somero de las series de la plara de Barcelona. Las principales conclusiones que extraemos son las siguientes. El precio de la movilidad del dinero es una categoría económica a tener en cuenta, ya que, sin duda, tuvo que influir en la economia financiera y en la economia real. En la primera debido a la fuerte volatilidad de los cambios, en la segunda por el efecto que las altas cotizaciones tuvieron sobre la comercializeción de los productos en el mercado interior.
\end{abstract}

\section{ABSTRACT}

In this note, we present a new point of view of how financial markets worked in ninetcenth century in Spain, focubaing on the price of money mobility, which is founded on the price of domeatic bills of exchange on eight days' aight. We reveal the cource, then the conceptual framework in the use of the course of the exchange as the price of money mobility. Finally, we present a brief analysis of the course of the exchangea in Barcelona. Some conclusions are pointed out: the price of money mobility is a pivotal concept, since there is no doubt it had to have significant effects over both financial

* Una primera versión de este trabajo se presentó en XI Simposio de Análisis Económico, celebrado en Barcelona en septiembre de 1986. Nos hemos decidido a publicarlo ante la insistencia de algunos compañeros de profesión, especialmente de Albert Carreras. Hemos tenido en cuenta algunas de las sugerencias que nos formularon Paco Comin, Pablo Martin Aceña, Carles Sudrià y Gabriel Tortella. 
and real economy. High volatility of exchanges affect the financial economy while high values of them affects the real economy.

Hace ya dos décadas diversos historiadores económicos realizaron una aportación muy sustantiva a nuestro conocimiento del sistema financiero español en el siglo xIx. Trabajos como los de Tortella (1970a) (1970b) (1973) (1974), Tedde (1974), Sánchez-Albornoz (1968a) (1968b) y Anes (1974) explicaron las características del sistema bancario y los rasgos principales de su evolución. Aquellos esfuerzos pioneros han tenido una continuidad limitada, salvando estudios tan meritorios como los de Nadal y Sudriá (1981), Martin Aceña (1981) (1985a) (1985b), Garcia López (1985) (1987) y Tedde (1988) 1. Actualmente aún sabemos muy poco sobre piezas tan fundamentales del sistema financiero como el mercado de capitales, o de la importancia de los servicios prestados por la banca o el papel jugado por los mercados de letras de cambio ${ }^{2}$.

Con esta nota pretendemos mostrar una perspectiva inédita del funcionamiento de los mercados financieros y contribuir con ello a su mejor conocimiento. Se trata de una primera reflexión sobre el precio de la transferencia de activos monetarios, basada en las cotizaciones de las letras de cambio a muy corto plazo. La nota consta de tres partes. En un primer y breve apartado se da cuenta de la fuente utilizada. En la segunda parte desarrollamos el marco conceptual que justifica considerar a los cambios como el precio de movilizar el dinero; asimismo, se expone la diversidad de elementos que los determinan. Por último, se procede a un análisis somero de las series de la plaza de Barcelona.

\section{LA FUENTE}

En la principales plazas mercantiles españolas la prensa decimonónica daba noticia regularmente de las cotizaciones de las letras (cambios) sobre otras plazas nacionales e internacionales. En algunos casos - como el de la capital

1 Esta relación no es, desde luego, exhaustiva. Solamente pretende señalar las aportaciones más relevantes e innovadoras.

2 Los autores reconocen su pequeña cuota de responsabilidad en el insuficiente desarrollo del estudio sobre el sistema financiero. Esperan que la cuota en cuestión se vea rebajada con la aparición de una obra, que estín preparando juntamente con J. Nadal, C. Sudrià, P. Pascual y A. Sánchez, en la cual se analiza la financiación de la industrialización y la evolución del sistema monetario en Cataluña entre 1815 y 1866 . Un sumario avance de resultados de esta investigación ha sido presentado en C. Sudrià, P. Pascual y L. Castañeda (1992). 
catalana - esta información se remonta a fines del siglo XVm ${ }^{3}$. Los datos procedían de los Corredores de Comercio. En cualquier caso, el Código de Comercio promulgado en 1829 establecia que los Colegios de Corredores debian llevar obligatoriamente un libro de precios de los activos reales y financieros en cuya venta intervenian los corredores. A partir de esa fecha, la prensa publicaba los precios que el Colegio establecia.

Las series que aqui presentamos han sido compiladas directamente de los libros de registro de cotizaciones del Colegio de Corredores Reales de Comercio de Barcelona, para el periodo 1830-1885. En lo que respecta a los años 1800-1829, hemos optado por calcular los cambios sobre la base de las operaciones asentadas en los libros de dos corredores que intervinieron en una gran número de transacciones de efectos. Hemos comprobado que las diferencias entre dichos cambios y los publicados en la prensa (Diario de Barcelona) son mínimas. Parece, por tanto, válido utilizar a ésta como fuente documental en trabajos similares referentes a otros centros mercantiles.

La fijación de la cotización oficial, desde 1830 , la realizaba el síndico con las notas sobre los precios a los que habian mediado los corredores durante la jornada. El proceso de cálculo concreto que realizaba el sindico se nos escapa, $y$, aunque cabe suponer que no era un procedimiento estadísticamente riguroso, las cotizaciones eran tomadas por los agentes económicos como el precio corriente de mercado 4.

\section{EL SIGNIFICADO DE LOS CAMBIOS $Y$ SUS DETERMINANTES}

Lo primero que hay que aclarar es que, entre plazas nacionales, las letras a la vista o a ocho días vista tenian una funcionalidad distinta a las de un vencimiento igual o superior a treinta dias. Los efectos a más corto plazo eran utilizados masivamente como medio de desplazar la liquidez o de realizar cobros y pagos a distancia. En este caso, el préstamo o adelanto de fondos era irrelevante. En suma, no se trataba propiamente de una operación de crédito, aunque quien tomaba el efecto asumía inevitablemente un riesgo. Los efectos (letras y

3 En ciertas ciudades este tipo de datos no aparecen en la prensa local hasta más tarde -incluso en la segunda mitad del siglo xax-, lo cual es un reflejo del desigual grado de desarrollo comercial, $y$, a la vez, de la paulatina integración del mercado financiero español.

- En la correspondencia de casas comerciales y empresas industriales encontramos que informaban a sus corresponsales del curso de los cambios copiándolos literalmente de la prensa o de los boletines de cotización de los corredores. 
pagarés) a medio y largo plazo, por contra, conllevaban siempre una transacción crediticia, que en muchas ocasiones la realizaban las entidades bancarias.

Una correcta comprensión de la evolución de los cambios requiere alguna explicación sobre el uso de las letras a muy corto vencimiento, así como de los términos empleados por los contemporáneos sobre el particular.

Toda letra constituye una orden de pago por una cantidad nominal consignada en el mismo documento, que el librado ha de hacer efectiva a su vencimiento en una plaza distinta a la de su creación. La cesión de la letra, a través de endoso, por parte del librador -0 del dador- a un nuevo tomador se identificaba como una operación de compraventa de activos monetarios situados en otra plaza. Al endosarse la letra, podia hacerse por una cantidad igual, superior o inferior a su importe. La diferencia entre el valor cobrado y el valor nominal, expresado como porcentaje respecto a este último, se denominaba precio del cambio, o, usualmente, cambio. Cuando el vendedor del efecto percibia una suma superior al valor nominal el cambio era con beneficio, y, viceversa, si se descontaba por una cantidad inferior el cambio se hacia con daño o pérdida. En el caso de ser igual el valor cobrado al nominal se decía que el cambio era a la par.

En los tratados de banca y comercio decimonónicos, asi como en los boletines de cotizaciones, y en cualquier medio mercantil, los cambios se clasificaban en nacionales y extranjeros. Estos últimos perduraron hasta bien entrado el presente siglo, habiendo sido reemplazados finalmente por los tipos de cambio entre divisas. Los cambios nacionales desaparecieron cuando el mercado monetario se unificó. En España, hasta la aparición de una entidad bancaria con implantación en todo el territorio nacional -el Banco de España-, habia tan sólo dos formas de rescatar los fondos liquidos situados en otros centros. Naturalmente, una de ellas consistía en el transporte físico del dinero. Alternativamente, podian ponerse en circulación letras a la vista $o$ a ocho días vista. En el caso especifico de Madrid y Barcelona - las plazas bursátiles-, podía recurrirse además a un activo semiliquido, la Deuda pública.

Dos sectores económicos eran los que, esencialmente, tenian necesidad de utilizar las letras a corto plazo. El primero lo formaban los industriales y productores en general en sus relaciones con proveedores y clientes de otras plazas. El fabricante solía reembolsarse el producto de sus ventas a través de letras que le eran enviadas por sus clientes para cobrarlas a un tercero. El, a su vez, compraba efectos para pagar a sus acreedores. El segundo grupo que actuaba en el mercado cambiario eran los comerciantes y financieros. Como es sabido, desarrollaban su actividad simultảneamente en varias plazas. Por consiguiente, les era preciso disponer de liquidez en diversos puntos a fin de que 
sus corresponsales pudiesen operar. En algunas ocasiones, comerciantes y banqueros necesitaban concentrar fondos, para lo cual realizaban los saldos acree. dores que mantenian con sus representantes. De igual modo que los productores, debian pagar un precio determinado para desplazar las disponibilidades liquidas. Así pues, en un mercado monetario no unificado los cambios reflejan el precio que estaban dispuestos a pagar los tenedores de activos monetarios por disponer inmediatamente de liquidez en una plaza comercial distinta de donde los tenían situados.

El precio por la movilidad del dinero estaba condicionado por el precio del dinero - lo que se entiende comúnmente como el tipo de descuento- y por los diferenciales en la cotización de la Deuda pública entre plazas '. Suponiendo un comportamiento racional de los agentes económicos, en el momento de reunir fondos líquidos en una plaza éstos tenian en cuenta las expectativas acerca de la variación futura de los cambios y el tipo de interés corriente. Cuando, por ejemplo, un individuo que dispusiese de liquidez en otros centros debia realizar un pago, optaría por tomar prestado en lugar de rescatar los fondos si esperaba que la variación favorable de los cambios fuese superior al interés pagado por el préstamo.

Por lo que respecta a la cotización de los valores públicos, al ser éstos activos semiliquidos, resultaban una alternativa a las letras a la vista como instrumento para desplazar la liquidez. Por ello, el diferencial en la cotización entre las plazas bursátiles tendía a corresponderse al tipo de cambio entre esas mismas poblaciones.

Aunque los factores que acabamos de mencionar influian en el precio, éste vino determinado en mayor medida por otros elementos. Según los manuales mercantiles de la época, los cambios reflejaban la balanza comercial entre las plazas ${ }^{6}$. Desde luego, se trata de una explicación limitada. En realidad, los cambios tendían en cada momento a traducir el saldo de la balanza básica. Por añadidura, en los precios a largo plazo concurrían los costes de transporte.

Por grande que fuese el saldo de la balanza básica entre dos ciudades, el coste de transporte y de custodia de la moneda metálica delimitaba, en épocas

S Para Barcelona disponemos de la serie de tipos de interés elaborada por Tortella (1973). A pesar de que esta serie guarda una relación débil con las que aquí presentamos, ambas son indicadores de la evolución del mercado monetario. Lo que sucede es que reflejan aspectos distintos del mismo.

Vale la pena llamar la atención sobre el hecho que el precio de la movilidad del dinero limitaba la eficacia de las subidas del tipo de interés para atraer fondos prestables del exterior de la propia plaza. La incertidumbre acerca de la evolución futura de los cambios tenía que representar un serio obstáculo para trasladarlos allí donde el interés era más alto.

- Véase, por ejemplo, Broussein (1805), Poy (1830), Guillén (1846), Castaño (1867). 
de normalidad, un umbral máximo para los cambios, tanto en las posiciones de daño como en las de beneficio (puntos oro). Durante las crisis, las necesidades apremiantes de liquidez rompian esa lógica, elevándose los cambios por encima del precio umbral de sustituibilidad de letras y acarrero de numerario. Como es harto conocido, los costes de transporte no permanecieron constantes a lo largo de la centuria. La tendencia secular fue descendente, como resultado de la progresiva mejora de la red de transportes. De todos modos, el efecto de la distancia sobre el precio de la movilidad del dinero no quedó eliminado hasta la constitución de una red bancaria nacional y la aparición de las transferencias bancarias entre cuentas corrientes. Estas condiciones no se dieron en nuestro pais hasta mediados de la década de 1880 . Con anterioridad, en la evolución del precio de la movilidad del dinero a corto plazo pueden distinguirse dos estados: las épocas de normalidad y los momentos de crisis.

Si ha quedado establecido que los costes de transporte del numerario determinaban el umbral de los precios máximos en las fases de normalidad, el signo y la tasa de éstos eran el resultado de la balanza básica entre unas y otras plazas. Teóricamente, tan sólo si la balanza entre dos ciudades estaba equilibrada, los cambios equivalían a los costes de transacción (gastos de negociación más riesgo). En otras palabras, el precio de la movilidad del dinero no es el coste de transacción. Cuando el saldo de las balanzas de pagos no era nulo, las cotizaciones de los cambios se movian de tal manera que en la plaza acreedora el daño se incrementaba, al mismo tiempo que en la plaza deudora disminuia, llegando a marcar posiciones de beneficio. Los cambios entre ciudades evolucionaban, por tanto, simétricamente. Hay que puntualizar que, para que se cumpliese completamente esta situación, los agentes tenian que disponer de información rápida sobre el estado de los restantes mercados de cambios, y, asimismo, poder transmitir con celeridad órdenes a los representantes que tenian emplazados en aquéllos. Estas condiciones no se dieron hasta que se contó con el telégrafo, que significó una mejora radical en las comunicaciones. En las plazas deudoras el descuento de las letras se hacía con beneficio, puesto que, al ser más numeroros los demandantes de papel que los oferentes, los primeros estaban dispuestos a tomar los efectos por un importe superior al nominal.

En las series que continuación presentamos destacan algunas puntas muy elevadas. Estos máximos respondian a dos fenómenos opuestos. Por una parte, los cambios más extremos reflejan grandes crisis económicas y financieras. Muy otro era el origen de algunas cotizaciones ciertamente altas que coincidían con momentos de reactivación económica. A nuestro entender, estas úl- 
timas expresan la incapacidad del sistema financiero para suministrar la liquidez necesaria a la economia real. Para suplir tal carencia, fabricantes y comerciantes debian retirar sus activos monetarios disponibles en otras plazas pagando precios considerables.

\section{ANALISIS DE LAS SERIES}

Hemos recogido los cambios máximos y mínimos mensuales sobre algunas plazas (Madrid, Cádiz, Zaragoza, Valencia, Santander, Valladolid, Reus y Mailaga). De entre ellas, las cinco primeras han sido elegidas como objeto de análisis en virtud de la continuidad de las series y de su importancia económica o su posición geográfica. En todos los casos, la marcha de los máximos y mínimos mensuales está muy estrechamente correlacionada 7. De hecho, tan sólo si hubiesen habido variaciones cíclicas de duración inferior a los dos meses no se habría producido tan elevada correlación entre los movimientos de los precios extremos. Es por ello que trabajamos únicamente con los cambios máximos mensuales, que tienen la ventaja respecto a los mínimos de hacer más clara. mente visibles las crisis de liquidez.

Antes de pasar a comentar la evidencia empirica reunida, advirtamos que se presentan los datos brutos, esto es, sin haber sido desestacionalizados ni sometidos a ningún tipo de manipulación estadistica. Finalmente, hay que señalar que, para mayor comodidad en la lectura de series y gráficos, hemos cambiado el signo de las mismas: los valores positivos corresponden a posiciones con daño - porcentaje de descuento-, y, viceversa, los valores negativos expresan el porcentaje de prima - posiciones de beneficio.

Observando las gráficas en conjunto destacan seis aspectos de gran interés. Primero, un comportamiento ciclico relativamente regular. La duración de la mayoría de los ciclos está comprendida entre 22 y 30 meses. De modo que las fluctuaciones de los cambios parecen estar en consonancia con los ciclos económicos de corta duración. La anterior observación se desprende sobre todo de los cambios sobre Madrid y Cádiz.

Segundo, el perfil de las grandes crisis se caracteriza por una escalada progresiva y un final brusco con caida repentina de las curvas. Una posible causa de este perfil podria residir en que, una vez superados los instantes más criti-

' El coeficiente de correlación de Pearson entre los precios máximos y minimos de Madrid es de 0,94 (sobre 947 casos). 
cos en las crisis de liquidez, era necesario reponer los fondos rescatados en las plazas donde se operaba comercialmente de forma más intensa.

Tercero, de los cinco mercados que hemos explorado, Madrid, Cádiz y Santander se mantuvieron sensibles a los problemas de liquidez y a la coyuntura económica hasta el último cuarto de la centuria. Por el contrario, los cambios sobre Valencia y Zaragoza se muestran menos fluctuantes desde los años 1850. Esto se explica por una más temprana reducción de los costes de accesibilidad. En conjunto, la persistencia de variaciones absolutas en las cotizaciones nos permite deducir que las letras a ocho dias vista fueron un instrumento monetario esencial hasta la década de 1880 .

Cuarto, todas las series evolucionaron de manera relativamente homogénea, como evidencian las altas correlaciones positivas entre ellas para el conjunto del periodo (véase cuadro 1). De dichas correlaciones se desprende como conclusión que las balanzas de pagos entre diferentes ciudades no se compensaban bilateralmente, sino multilateralmente.

Quinto, la balanza de pagos de Barcelona en relación al conjunto peninsular fue siempre favorable, salvo en muy contadas coyunturas. Queda demostrada esta afirmación al observar que los cambios fueron casi siempre con daño y a un nivel de cotización superior a lo que razonablemente cabe suponer que fueron los costes de transacción.

Sexto y último, la cronología de las grandes crisis pone de manifiesto la conexión de Barcelona con la economia internacional.

La evolución secular de los cambios puede dividirse en tres grandes periodos: 1) de 1800 a $1828-29$; 2) de 1829 a 1850 ; 3) de 1850 a 1885 . A partir de esta última fecha, los cambios permanecieron fijos de forma indefinida.

Los años comprendidos entre el comienzo del siglo y finales de la tercera década estuvieron marcados por una tensión constante, como lo muestra la intensidad y frecuencia de cambios muy elevados. Para esta primera etapa, algunas de las correlaciones obtenidas son inferiores a la etapa siguiente. Atribuimos hipotéticamente este hecho a que los costes de información eran mayores antes de 1830.

Los primeros máximos de aquel período son los de 1802 y 1805 . En la primera fecha se aprovechó la paz con Inglaterra para repatriar grandes masas de capitales. En consecuencia, la sobreoferta de papel presionó a una negociación con fuerte daño. Tres años más tarde, la guerra y el colapso del tráfico marítimo, unido a los apuros de la Hacienda, dieron lugar a una aguda crisis.

Después del paréntesis de la Guerra de Independencia, se suceden persistentes puntas entre 1815 y 1817 , que podrían obedecer a causas diversas: caos monetario, descapitalización del país y desarticulación del sistema financiero. 
De 1820 a 1823 la inestabilidad política se tradujo en notables oscilaciones en el precio de la movilidad del dinero, que culminan en junio de 1823. En 1827 los cambios vuelven a marcar tipos elevados, en sintonía con la crisis económica internacional.

El segundo gran periodo, que abarca de $1828-29$ a 1850 , aparece como una época de transición. En su transcurso se registran dos puntas cíclicas máximas, que tienen por origen las crisis económicas internacionales de $1837 \mathrm{y}$ 1847-48. Plausiblemente, el valor tan extremadamente elevado de esta última se deba, por un lado, a la suspensión del descuento comercial por el Banco de Barcelona, que provocó una situación delicadísima en el mundo de los negocios barcelonés; y, por otro, a la desconfianza del público sobre la solidez de los billetes de los bancos de emisión en Madrid. Durante la primera década de este periodo, en los años treinta, las convulsiones políticas y la guerra civil volvieron a influir poderosamente en la marcha de los cambios. Un comentario de especial significación merecen los altos valores de 1840-41, simultáneos con un fuerte relanzamiento de la economía catalana. Como ya se ha señalado en el epigrafe anterior, creemos que no es desacertado culpar al atrasado sistema financiero de la inelasticidad de la oferta monetaria.

El último gran periodo va de 1850 a 1885 . La tónica general dentro de él fue la de una mayor estabilidad y tipos más bajos. Es de destacar que durante los años $1855-57$ se dieron sostenidas posiciones con beneficio. Moviéndonos nuevamente en el terreno de las hipótesis, esta anómala posición deudora de Barcelona con el resto de las plazas estaria motivada por un serio deterioro de los términos de intercambio entre los precios de los productos manufacturados y los agrarios. Recuérdese que fueron años de fuerte alza de los precios agrarios, fruto de la combinación de crisis de subsistencias y la coyuntura de la Guerra de Crimea. Ya en la década siguiente, encontramos la punta de 1861 provocada por el «hambre de algodón». Sobresale a continuación la crisis económica global de 1864-66, que dibuja la última gran curva alcista, con un máximo espectacular en las principales plazas. La guerra carlista y, en el caso de Madrid, el arreglo de la Deuda de Salaverría provocaron nuevamente sensibles tensiones en los cambios. El último movimiento de las series se produjo en 1882 , a raiz del colapso bursátil.

Los cambios interiores o nacionales dejaron de tener relevancia económica con el establecimiento por el Banco de España en 1885 del servicio de transferencias entre cuentas de diferentes sucursales. En ese momento el Banco ya contaba con una red de sucursales lo suficientemente densa para que este nuevo servicio pudiese ser ampliamente aprovechado en sustitución de las letras de cambio a muy corto plazo. Esta innovación financiera tuvo que repre- 


\section{CUADRO 1}

Correlaciones entre las series de los cambios de Madrid, Cádiz, Valencia, Zaragoza y Santander

\begin{tabular}{|c|c|c|c|c|c|c|}
\hline & & Madrid & Cadiz & Valencia & Zaragoze & Saneander \\
\hline \multirow[t]{4}{*}{ Madrid } & $\begin{array}{l}1800-1855 \text { «m } \ldots \ldots \ldots \\
n .^{\circ} \text { Casos } \ldots \ldots \ldots \ldots \ldots\end{array}$ & 1 & $\begin{array}{l}.420 \\
(896)\end{array}$ & $\begin{array}{r}.327 \\
(844)\end{array}$ & $\begin{array}{l}.461 \\
(743)\end{array}$ & $\begin{array}{l}.685 \\
(513)\end{array}$ \\
\hline & $\begin{array}{l}1800-1829 \text { «m } \ldots \ldots \ldots \\
n^{\circ} \text { casos } \ldots \ldots \ldots \ldots \ldots\end{array}$ & 1 & $\begin{array}{l}.618 \\
(238)\end{array}$ & $\begin{array}{r}.376 \\
(190)\end{array}$ & $\begin{array}{l}.312 \\
(103)\end{array}$ & - \\
\hline & $\begin{array}{l}1830-1850 \text { ars } \ldots \ldots \ldots \\
n^{\circ} \text { casos } \ldots \ldots \ldots \ldots \ldots\end{array}$ & 1 & $\begin{array}{l}.431 \\
(241)\end{array}$ & $\begin{array}{l}.415 \\
(244)\end{array}$ & $\begin{array}{l}.513 \\
(223)\end{array}$ & $\begin{array}{l}.715 \\
(134)\end{array}$ \\
\hline & $\begin{array}{l}1851.1885 \text { ar } \ldots \ldots \ldots \\
\mathbf{n}^{\circ} \cos 08 \ldots \ldots \ldots \ldots\end{array}$ & 1 & $\begin{array}{l}.466 \\
(417)\end{array}$ & $\begin{array}{r}.242 \\
(410)\end{array}$ & $\begin{array}{l}.157 \\
(417)\end{array}$ & $\begin{array}{l}517 \\
(379)\end{array}$ \\
\hline \multirow[t]{4}{*}{ Cidiz } & $\begin{array}{l}1800-1855 \text { ars } \ldots \ldots \ldots \\
\text { n. } .^{\circ} \text { casos } \ldots \ldots \ldots \ldots \ldots . . . . .\end{array}$ & & 1 & $\begin{array}{l}541 \\
(818)\end{array}$ & $\begin{array}{l}.332 \\
(729)\end{array}$ & $\begin{array}{l}.360 \\
(510)\end{array}$ \\
\hline & $\begin{array}{l}1800-1829 \text { ars } \ldots \ldots \ldots \\
\text { n. }{ }^{\circ} \text { casos } \ldots \ldots \ldots \ldots \ldots\end{array}$ & & 1 & $\begin{array}{l}.373 \\
(168)\end{array}$ & $\begin{array}{l}.375 \\
(90)\end{array}$ & - \\
\hline & $\begin{array}{l}1830-1850 \\
\mathrm{n}^{\circ} \cos 0 \mathrm{~s} \ldots \ldots \ldots \ldots \ldots\end{array}$ & & 1 & $\begin{array}{l}.264 \\
(240)\end{array}$ & $\begin{array}{l}.287 \\
(222)\end{array}$ & $\begin{array}{l}.362 \\
(131)\end{array}$ \\
\hline & $\begin{array}{l}1851-1885 \text { wr } \ldots \ldots \ldots \\
n^{\circ} \text { casos } \ldots \ldots \ldots \ldots \ldots\end{array}$ & & 1 & $\begin{array}{l}.365 \\
(410)\end{array}$ & $\begin{array}{l}.190 \\
(417)\end{array}$ & $\begin{array}{l}.360 \\
(379)\end{array}$ \\
\hline \multirow[t]{6}{*}{ Valencia } & $\begin{array}{l}1800-1855 \text { «r } \ldots \ldots \ldots \\
n^{\circ} \text { cesos } \ldots \ldots \ldots \ldots \ldots\end{array}$ & & & 1 & $\begin{array}{l}.485 \\
(706)\end{array}$ & $\begin{array}{l}.470 \\
(508)\end{array}$ \\
\hline & $1800-1829$ ar $\ldots \ldots \ldots$ & & & 1 & 221 & - \\
\hline & $1830-1850$ «г $\ldots \ldots \ldots$ & & & 1 & .349 & .381 \\
\hline & $\mathrm{n}^{\circ} \mathrm{casos} \ldots \ldots \ldots \ldots$ & & & & $(222)$ & (134) \\
\hline & $1851-1885$. $\ldots \ldots \ldots$ & & & 1 & .546 & .391 \\
\hline & $\mathrm{n}^{\circ} \operatorname{casos} \ldots \ldots \ldots \ldots \ldots$ & & & & (411) & (374) \\
\hline \multirow[t]{6}{*}{ Zaragoza } & $\begin{array}{l}1800-1855 \text { «m } \ldots \ldots \ldots \\
n^{\circ} \text { casos } \ldots \ldots \ldots \ldots \ldots\end{array}$ & & & & 1 & $\begin{array}{l}.623 \\
(507)\end{array}$ \\
\hline & $1800-1829 * \mathrm{~m} \times \ldots \ldots$ & & & & 1 & - \\
\hline & n. ${ }^{\circ}$ casos............... & & & & & \\
\hline & $1830-1850$ ars ......... & & & & 1 & $\begin{array}{l}.693 \\
(127)\end{array}$ \\
\hline & $\begin{array}{l}n . c 2 s 0 s \ldots \ldots \ldots \ldots \\
1851.1885 \text { ars } \ldots \ldots \ldots\end{array}$ & & & & 1 & .383 \\
\hline & n. ${ }^{\circ}$ casos $\ldots \ldots \ldots \ldots \ldots$ & & & & & (380) \\
\hline \multirow[t]{4}{*}{ Santander } & $1800-1855$ «ro....... & & & & & 1 \\
\hline & $\begin{array}{l}\mathrm{n}^{\circ} \operatorname{css}(\ldots \ldots \ldots \ldots \ldots \\
1800-1829 \text { ars } \ldots \ldots \ldots\end{array}$ & & & & & 1 \\
\hline & $\begin{array}{l}n^{\circ} \text { cssos } \ldots \ldots \ldots \ldots \ldots \\
1830-1850\end{array}$ & & & & & 1 \\
\hline & $\begin{array}{l}n^{\circ} \text { casos } \ldots \ldots \ldots \ldots \ldots \\
1851-1885 \text { «m } \ldots \ldots \ldots \\
n^{\circ} \text { casos } \ldots \ldots \ldots \ldots \ldots\end{array}$ & & & & & 1 \\
\hline
\end{tabular}


sentar una sensible disminución de los costes de transacción en la movilidad geográfica del dinero, lo que explica su inmediata aceptación. Podemos decir que sólo desde entonces España, además de tener una única moneda, tenia también un sistema monetario integrado.

\section{CONCLUSIONES}

En estas notas hemos pretendido dar a conocer un nuevo indicador para el estudio de la historia financiera española, los cambios entre plazas nacionales de las letras a ocho días vista.

La variable, y la fuente, tienen dos grandes virtualidades. Una es que nos permiten tener un conocimiento preciso sobre la evolución coyuntural de los mercados monetarios (en plural, puesto que no había un mercado nacional único). La segunda consiste en que a través de un mismo indicador podemos observar todas las coyunturas que se han sucedido a lo largo de casi un siglo. Ante la ausencia de series financieras con estas características, las que aqui sugerimos utilizar adquieren una relevancia especial.

El precio de la movilidad del dinero es una categoría económica a tener en cuenta, ya que influyó indudablemente en la economía financiera y en la economía real. La fuerte volatilidad de los cambios incrementaba el riesgo en los movimientos de capital. Por otra parte, las altas, y, en ocasiones sostenidas, cotas alcanzadas por las cotizaciones tuvieron que afectar a la comercialización de los productos en el mercado interior. Al precio de las mercancías debía añadírsele el precio de la movilidad del dinero.

Una última conclusión, que es al tiempo una propuesta de trabajo, se hace evidente. Deberia llegar a confeccionarse un mapa de los cambios de cada plaza con respecto a las restantes. Con ello obtendriamos una mejor comprensión de la dinámica de la vida mercantil local y regional, y, a la vez, de la red de ciudades. 


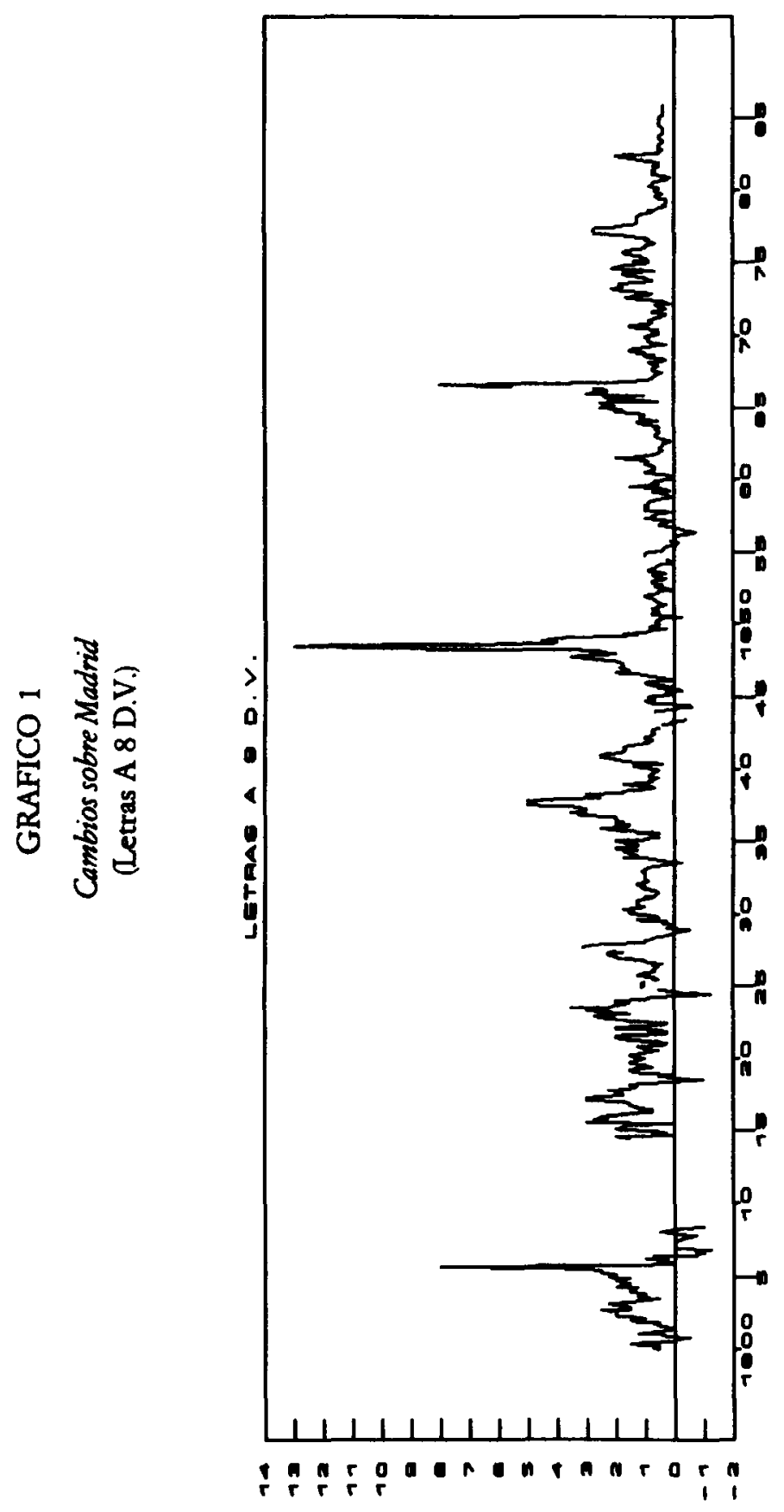




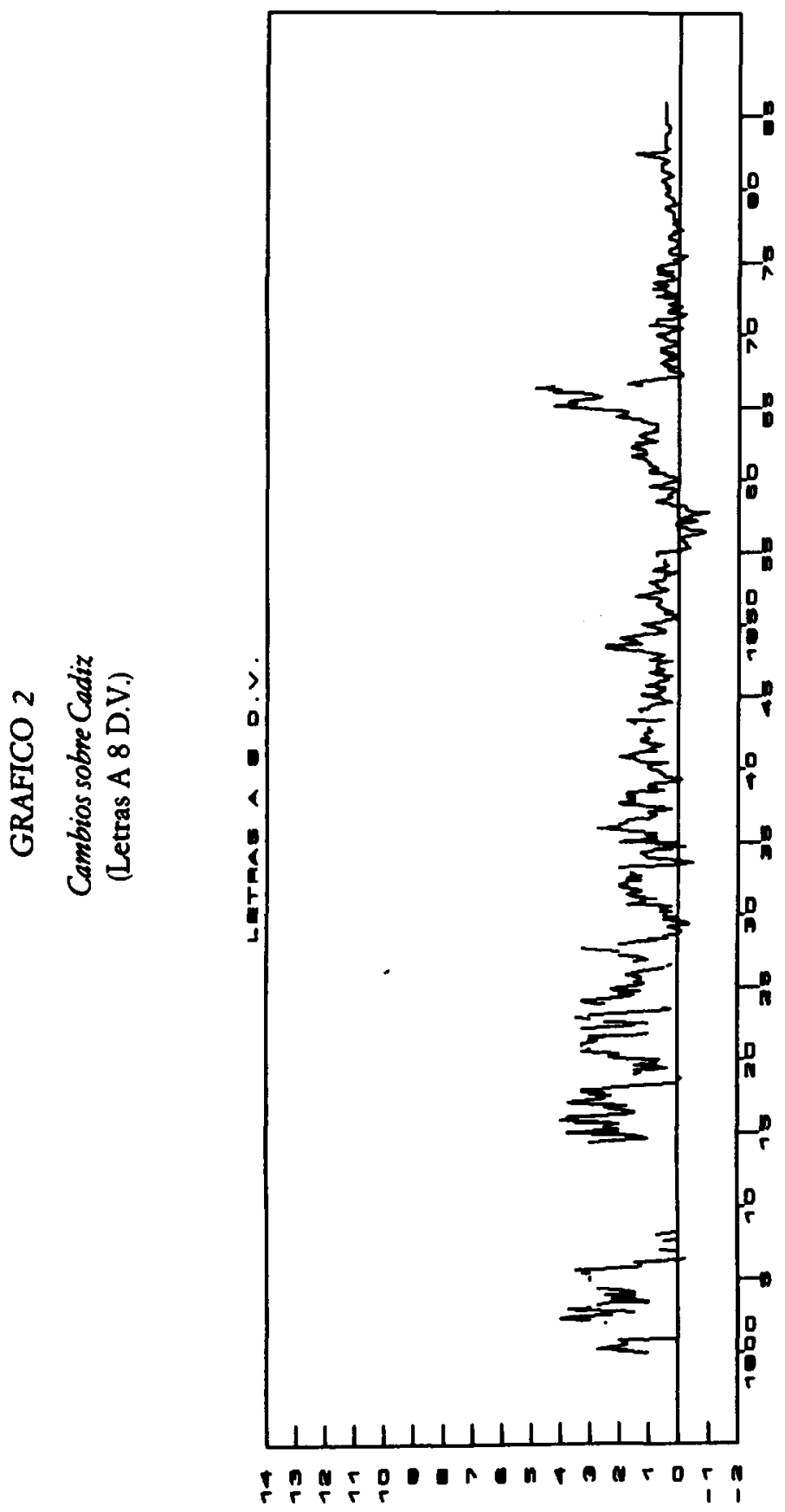




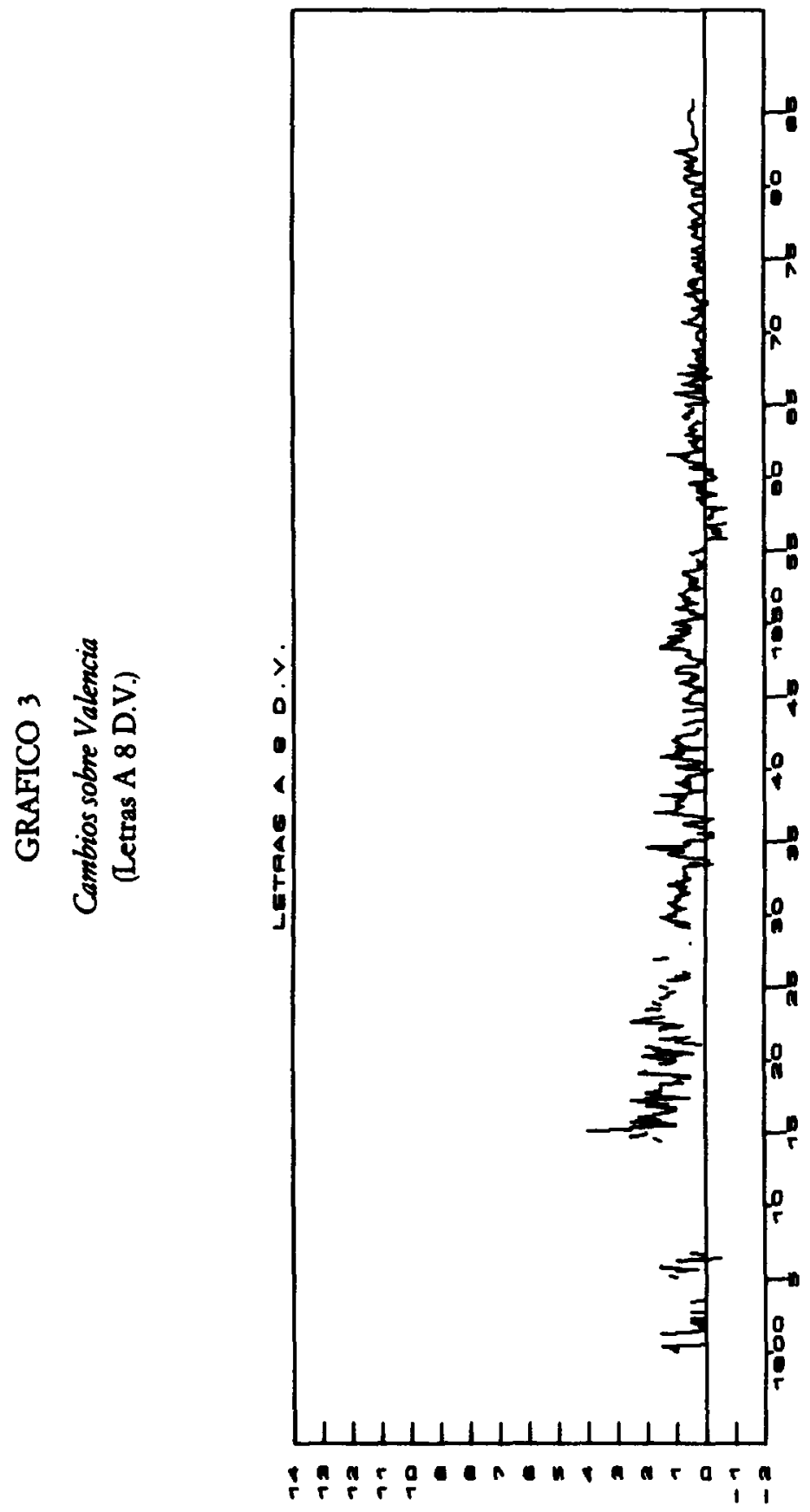




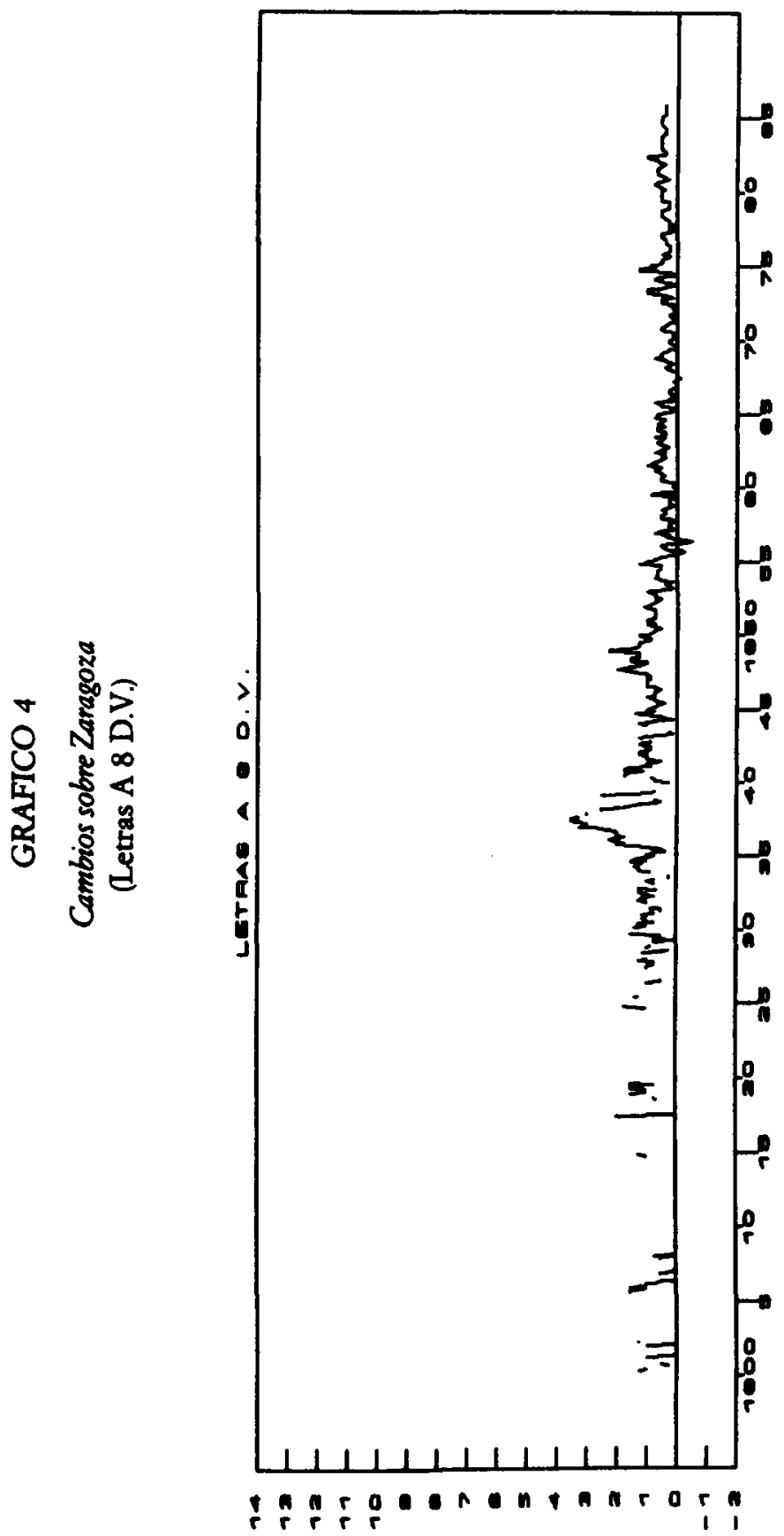




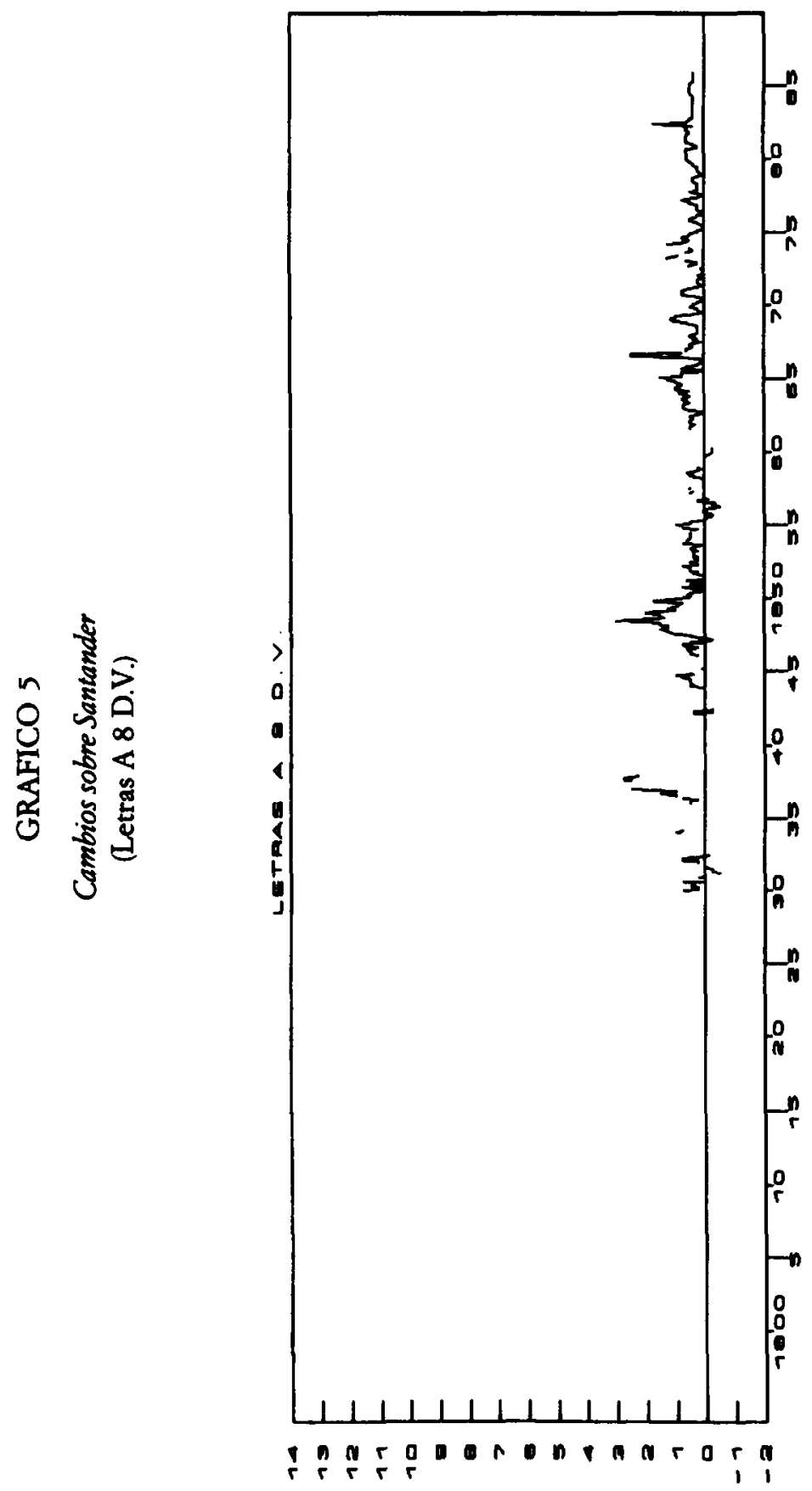




\section{BIBLIOGRAFIA CITADA}

ANES, Rafael (1974): «El Banco de España, 1874-1914: un banco nacional», en Schwartz, Pedro (ed.), La banca española en la Restauración, tomo I, Política y finanzas, Madrid, Servicio de Estudios del Banco de España.

Broussein, Martín (1805): La ciencia del giro, Madrid, s.p.j.

CASTANO, Francisco (1867): Guía manual del comercio y de la banca, o sea tratado completo teórico-práctico de operaciones mercantiles y de monedas, pesas y medidas, nacionales y extranjeras. 5.' edición, Alicante, Imp. y Lib. Vda. de Ibarra.

GARCta LOPEZ, José Ramón (1985): «Banqueros y comerciantes banqueros, clave oculta del funcionamiento del sistema bancario español del s. XIX», Moneda y Crédito, n. ${ }^{\circ}$ 175, pp. 59-85.

- (1987): Los comerciantes banqueros en el sistema bancario español: Estudio de casas de banca asturianas en el siglo xIx, Oviedo, Universidad de Oviedo.

GUILLEN SUAREZ, Antonio (1846): El verdadero cambista teórico y práctico compilado y metodizado muy detenidamente, Barcelona, Imp. Pons y Cia.

MARTIN ACENA, Pablo (1981): «España y el patrón-oro 1880-1913», Hacienda Pública Españole, n. 69 , pp. $267-290$.

- (1985a): «Desarrollo y modernización del sistema financiero, 1844-1935», en SAnCHEZ-AlboRNoz, N. (ed.), La modemización económica de España, Madrid, Alianza.

- (1985b): «La politica monetaria durante la Restauración, 1875-1914», en GARC1^ Delgado, J. L. (ed.), La España de la Restauración, Madrid, Siglo XXI.

NADAL, Jordi, y SudruA, Carles (1981): Història de la Caixa de Pensions, Barcelona, Edicions 62.

Poy COMEs, Manuel (1830): Tratado general de cambios, usos y estilos sobre el pago de las letras, monedas, pesas y medidas de todas las naciones comerciantes y en particular de España, Barcelona, Imp. Hnos. Juan y Jaime Gaspar.

SANCHEZ-AlBORNOZ, Nicolás (1968a): "Los bancos y las sociedades de crédito en las provincias, 1856-1868», Moneda y Crédito, n. ${ }^{\circ} 104$, pp. 39-68.

- (1968b): España bace un siglo: una economia duah Barcelona, Peninsula.

Sudru, Carles; Pascunl, Pere, y CastaNeda, Lluís (1992): «Oferta monetaria y financiación industrial en Cataluña, 1815.1860m, Revista de Historia Industriah n. ${ }^{\circ}$ 1, pp. 189.202.

TEDDE DE LORCA, Pedro (1974): «La banca privada española durante la Restauración, 1874-1914", en SchwarTz, P. (ed.), La Banca española en la Restauración, tomo I, Politica y finanzas, Madrid, Servicio de Estudios del Banco de España.

- (1988): El Banco de San Carlos, Madrid, Banco de España/Alianza Editorial.

Tortella Casares, Gabriel (1970a): «El Banco de España entre 1829 y 1929. La formación de un banco central», en El Banco de España. Una historia económica, Madrid, Banco de España.

- (1970b): «La evolución del sistema financiero de 1856 a 1868*, en Ensayos sobre la economía española a mediados del siglo xIX, Madrid, Ariel.

- (1973): Los orígenes del capitalismo en España, Madrid, Tecnos.

- (1974): «Las magnitudes monetarias y sus determinantesw, en SCHwarTZ, P. (ed.), La Banca española en la Restauración, tomo I, Política y finanzas, Madrid, Servicio de Estudios del Banco de España. 